\title{
Orofacial Infections in Kenya: A Retrospective Study
}

\author{
Kimathi Denis Mutwiri, ${ }^{1}$ Elizabeth Dimba, ${ }^{2}$ Bernard Mua Nzioka ${ }^{3}$ \\ ${ }^{1}$ School of Dental Sciences, University of Nairobi \\ ${ }^{2}$ Department of Oral and Maxillofacial Surgery, Oral Pathology and Oral Medicine, School of Dental \\ Sciences, University of Nairobi \\ ${ }^{3}$ Department of Periodontology/Community and Preventive Dentistry, School of Dental Sciences, \\ University of Nairobi
}

Correspondence to: Mr. Kimathi Denis Mutwiri; email: deniskim371@gmail.com

Received: 09 January 2020; Revised: 13 May 2020; Accepted: 29 May 2020; Available online: 21 June 2020

\begin{abstract}
Background: Orofacial infections are either odontogenic or non-odontogenic in nature. The clinical spectrum of these infections is diverse. This study aimed to describe the presentation and management of patients presenting with orofacial infections at Kenyatta National Hospital (KNH). Materials and Methods: This was a retrospective study based on clinical records of patients treated for orofacial bacterial infections at $\mathrm{KNH}$ from January 2016 to December 2018. Data on the following study variables were obtained and analyzed: demographic data, clinical presentation, diagnosis, management, and treatment outcome. Results: 214 clinical records were studied. Male to female ratio was 1.4:1 with age range of 3 months to 78 years (mean $=27.0$ years). Swelling $(96.30 \%, n=206)$ was the most common symptom, next was pain $(58.90 \%, n=123)$. The most common source of infection was odontogenic $(60.30 \%$, $n=129)$ in nature. Permanent teeth $(57.00 \%, n=122)$ were more commonly involved than deciduous teeth $(2.80 \%, n=6)$. In both dentitions, the mandibular posterior teeth were the most commonly involved, Ludwig's angina $(30.84 \%, n=66)$ and submandibular
\end{abstract}

abscess $(25.23 \%, n=54)$ were the most common clinical diagnoses of orofacial bacterial infection. The commonly used treatment modality was a triad of extraction of the associated tooth, incision and drainage, and antibiotic therapy. These management modalities resulted in favorable treatment outcomes $(92.50 \%$, $n=198$ ) in most cases. Conclusion: Orofacial infections can occur among all sociodemographic groups. These infections are potentially life-threatening if not diagnosed early and managed promptly. Multidisciplinary teams are required to manage the severe morbidity and mortality of advanced orofacial infections.

Keywords: Orofacial infections, Odontogenic, Abscess, Ludwig's angina

Ann Afr Surg. 2021; 18(1): 45-51

DOI: http://dx.doi.org/10.4314/aas.v18i1.9

Conflicts of Interest: None

Funding: None

(C) 2021 Author. This work is licensed under the Creative Commons Attribution 4.0 International License.

\section{Introduction}

Orofacial infections are a common presenting complaint in medical and dental offices, and sometimes, in severe cases, hospital emergency departments. Infections of the mouth and associated structures can be classified as

odontogenic and non-odontogenic in nature (1). Odontogenic infections originate within the tooth or associated fungi or viruses and are characteristically caused by bacteria. Mucosal infections by bacteria 
account for most of the oral non-odontogenic infections (2). The primary causes of orofacial infections are nonvital teeth, pericoronitis, dental procedures, periodontal disease and infected cysts. Rarer causes include trauma, salivary gland and lymph node lesions (3).

The clinical spectrum of orofacial infections is diverse, ranging from localized and indolent conditions to lifethreatening conditions. Once established, the pathophysiological course of a given infectious process varies. This depends on the virulence and number of organisms, host resistance and the local anatomy of the involved area (1). Local anatomy is an important factor as infection spreads through tissue planes in the path of least resistance, through blood or lymphatics. Spread of infection in the orofacial region may lead to airway obstruction, septicemia or intracranial spread (3). The morbidity and mortality related to these orofacial infections depend on the site of involvement and the degree of spread to the other tissues (4).

Accurate diagnosis of orofacial infections is important for timely treatment and for public health management. It is therefore important that health care workers are fully aware of the sociodemographic characteristics of patients presenting with orofacial infections, presentations of various orofacial bacterial infections, teeth and anatomical sites frequently involved for timely and appropriate management of these patients to prevent life-threatening complications associated with these infections.

No studies are known investigating the pattern of orofacial infections in the Kenyan population. The aim of this study was to describe the presentation and management of patients presenting with orofacial infections at Kenyatta National Hospital (KNH). Information from this study will help in the management of cases and provide data for future planning of treatment protocols.

\section{Methods}

The study setting is a specialized maxillofacial unit in a tertiary referral hospital. This was a retrospective study covering the period between January 2016 and December 2018. Ethical approval to carry out this research was obtained from the University of Nairobi and the National Hospital Ethics Research and Standards Committee (Approval numberUP103/02/2019). Permission to use patients' records was sought from the relevant authorities at Kenyatta National Hospital.

Convenience sampling was used to retrieve clinical records of patients treated for orofacial infections at $\mathrm{KNH}$ during the study period.

Data on the following study variables were obtained using data collection forms and analyzed: demographic data, clinical presentation, diagnosis, management and treatment outcome (discharge or death).

Analysis used SPSS version 23 and Microsoft Excel 2013. Frequency tables were used to present data.

\section{Results}

Three hundred clinical records were identified for review: 226 clinical records were retrieved and 74 were missing. Of the 226 clinical records retrieved, 12 had incomplete data and were excluded from the study. 214 clinical records were therefore included in the analysis.

\section{Sociodemographic characteristics}

Two hundred and fourteen patient records were reviewed: 127 male (59.3\%) and 87 female (40.7\%), age range 3 months to 79 years, with a mean age of 27.0 years. The modal ages for patients with orofacial bacterial infections were between 20 and 29 years $(39.46 \%)$. Most patients had informal employment $(33.6 \%, n=72)$ or were dependents $(31.8 \%, n=38) ; 38$ patients $(17.8 \%)$ were formally employed.

\section{Presenting symptoms}

Swelling $(96.3 \%, n=206)$ and pain $(58.9 \%, n=123)$ were the most common symptoms. Other symptoms with their distribution are given in Table 1.

\section{Source of orofacial bacterial infection}

The most common source of infection was odontogenic in nature $(60.3 \%, n=129)$, next was non-odontogenic infection $(20.6 \%, n=44)$ while the source was unspecified or not known in 41 patients $(n=19.2 \%)$. 
MUTWIRI ET AL.

Table 1: Presenting symptoms of the patients seen

\begin{tabular}{ll}
\hline Symptom & No. of cases $(\%)$ \\
\hline Swelling & $206(96.3)$ \\
Pain & $126(58.9)$ \\
Trismus & $89(41.6)$ \\
Tenderness & $68(31.8)$ \\
Purulent discharge & $39(18.2)$ \\
Respiratory distress & $38(17.8)$ \\
Dysphagia & $31(14.5)$ \\
Fever & $27(12.6)$ \\
Elevated tongue & $26(12.1)$ \\
\hline
\end{tabular}

\section{Fascial space involved in the infectious process}

The fascial space most commonly involved was submandibular space $(89.60 \%, n=147)$. Other fascial spaces were involved as follows: buccal space $(15.90 \%$, $n=26)$, masticator space $(7.9 \%, n=13)$ and anterior visceral $(1.8 \%, n=3)$. Infratemporal space was the least involved $(0.6 \%, n=1)$.

\section{Teeth associated with the infection}

Permanent teeth were the most commonly involved teeth $(57.0 \%, n=122)$ and deciduous teeth the least common source of infection $(2.8 \%, n=6)$. The teeth associated with the infection were not specified in $17.3 \%(n=37)$ of the cases. Of the permanent teeth, mandibular posterior teeth were the most commonly involved teeth $(34.1 \%, n=73)$, while mandibular and maxillary anterior teeth were the least involved $(0.5 \%$, $n=1$ ) (Table 2).

\section{Diagnosis of orofacial bacterial infections}

Ludwig's angina was the most common clinical diagnosis $(30.84 \%, n=66)$, next was submandibular abscess $(25.23 \%, n=54)$ [Table 3]. The sequelae of odontogenic infections was variable. Although the first area of spread occurred in the contiguous tissues, deciduous and permanent dentitions did not have clear patterns of spread into fascial spaces. Likewise, individual teeth exhibited varying patterns of anatomical spread.

The diagnoses described in Table 3 were as per the patients' records. No attempt was made to audit the accuracy of these diagnoses retrospectively.
Table 2: Teeth involved in the infectious process

\begin{tabular}{lc}
\hline Teeth & Frequency (\%) \\
\hline Permanent teeth & $1(0.5)$ \\
Mandibular anterior teeth & $73(34.1)$ \\
Mandibular posterior teeth & $1(0.5)$ \\
Maxillary anterior teeth & $9(4.2)$ \\
Maxillary posterior teeth & $5(2.3)$ \\
Multiple teeth & $54(25.2)$ \\
Unspecified & $71(33.2)$ \\
None & $214(100)$ \\
Total & \\
Deciduous teeth & $1(0.5)$ \\
Maxillary anterior tooth & $4(1.9)$ \\
Mandibular posterior & $36(16.8)$ \\
Unspecified & $173(80.8)$ \\
None & $214(100)$ \\
Total &
\end{tabular}

Table 3: Clinical diagnosis of orofacial bacterial infections

\begin{tabular}{lc}
\hline Clinical diagnosis & Frequency $(\%)$ \\
\hline Ludwig's angina & $66(30.84)$ \\
Submandibular abscess & $54(25.23)$ \\
Orbital cellulitis & $13(6.07)$ \\
Orbital abscess & $10(4.67)$ \\
Dentoalveolar abscess & $9(4.21)$ \\
Necrotizing fasciitis & $8(3.74)$ \\
Buccal abscess & $6(2.80)$ \\
Surgical site infection & $5(2.34)$ \\
Masticator space abscess & $4(1.87)$ \\
Others & $39(18.22)$ \\
Total & $214(100.00)$ \\
\hline
\end{tabular}

\section{Treatment modality used}

Pharmacologic treatment modality

A combination of antibiotics and analgesics was the most common treatment regimen $(91.6 \%, n=196)$. Amoxicillin-clavulanic acid $(45.3 \%, \quad n=96)$ and Ceftriaxone $(39.6 \%, n=84)$ were the most commonly prescribed antibiotics singly while metronidazole (75.9\%, $n=161)$ was the most commonly prescribed antibiotic in combination

The most commonly prescribed analgesic was paracetamol $(51.5 \%, n=102)$, next were non-steroidal anti-inflammatory drugs [diclofenac $(43.9 \%, n=87)$ and Ibuprofen $(18.7 \%, n=37)]$ and tramadol $(17.2 \%, n=34)$. Other pharmacologic intervention provided was dexamethasone in $62.7 \%(n=74)$ of the patients, and an antiseptic mouthwash in $29.7 \% \quad(n=35)$. Antibiotic 
combinations used in different clinical situations overlapped considerably as per best clinical practice guidelines for orofacial infections of bacterial origin (Table 4).

Table 4: Antibiotic regimen prescribed

\begin{tabular}{lc}
\hline Antibiotic regimen & Frequency (\%) \\
\hline Amoxicillin-clavulanic acid, & $46(21.5)$ \\
metronidazole & $38(17.8)$ \\
Ceftriaxone, metronidazole & $17(7.9)$ \\
Amoxicillin-clavulanic acid & $7(3.3)$ \\
Clindamycin & \\
Amoxicillin-clavulanic acid, & $6(2.8)$ \\
$\quad$ ceftriaxone, metronidazole & $100(46.3)$ \\
Others & $214(100.00)$ \\
Total & \\
\hline
\end{tabular}

\section{Surgical treatment modality}

Table 5 shows $62.1 \%(n=172)$ of patients underwent incision and drainage, $28.2 \%(n=78)$ had the offending tooth extracted and $6.5 \%(n=18)$ underwent surgical debridement.

Table 5: Surgical intervention frequencies.

\begin{tabular}{lcc}
\hline Surgical intervention & \multicolumn{2}{c}{ Responses } \\
frequencies & $n=277$ & $\%$ \\
\hline Incision and drainage & 172 & 62.1 \\
Extraction & 78 & 28.2 \\
Debridement & 18 & 6.5 \\
Tracheostomy & 4 & 1.4 \\
Skin grafting & 2 & 0.7 \\
Dry socket curettage & 1 & 0.4 \\
FESS for Parasinusitis & 1 & 0.4 \\
Surgical exploration & 1 & 0.4 \\
Total & 277 & 100.0 \\
\hline
\end{tabular}

\section{Other treatment modality}

Mouth-opening exercises were carried out in $4.7 \%$ $(n=10)$ of the patients while endodontic therapy was given to $0.9 \%(n=2)$.

\section{Treatment outcome}

Treatment outcomes were favorable with complete resolution of infection in $92.5 \%(n=198)$ of patients while $7.00 \%(n=15)$ died and $0.5 \%(n=1)$ developed complication of blindness in the right eye from orbital cellulitis. Treatment outcomes were influenced by patients' medical comorbidity. The relationship between patients' medical comorbidity and treatment outcome was statistically significant $(\mathrm{p}=0.000)$.

Most patients $(83.4 \%, n=181)$ did not have a medical comorbidity on presentation. Of those with a medical comorbidity, diabetes mellitus was the most common $(4.1 \%, n=9)$, next was hypertension $(2.8 \%, n=6)$ (Table $6)$.

Table 6: Relationship between patients' treatment outcome and comorbidity

\begin{tabular}{lccc}
\hline Comorbidities & \multicolumn{3}{c}{ Treatment outcome } \\
\cline { 2 - 4 } & Discharged & Death & Total \\
\hline None & 177 & 4 & 181 \\
Diabetes mellitus & 2 & 7 & 9 \\
HIV and AIDS & 6 & 1 & 7 \\
Hypertension & 3 & 3 & 6 \\
Asthma & 4 & 0 & 4 \\
Epilepsy & 2 & 0 & 2 \\
Acute kidney injury & 0 & 1 & 1 \\
Acute myeloid & 0 & 1 & 1 \\
leukemia & & & \\
Adenoids & 1 & 0 & 1 \\
Cerebral palsy & 1 & 0 & 1 \\
Rhabdomyosarcoma & 1 & 0 & 1 \\
Rickets & 1 & 0 & 1 \\
Sickle cell anemia & 1 & 0 & 1 \\
Tuberculosis & 0 & 1 & 1 \\
Total & 199 & 15 & 214 \\
& & $\chi^{2}=0.000$ & \\
\hline
\end{tabular}

\section{Discussion}

Orofacial bacterial infections, which are caused by a range of diseases including caries, periodontal diseases, trauma and complications of treatment, can affect all sociodemographic groups (5). This was observed in this study where infections involved patients aged between 3 months and 78 years and among males and females, which was in agreement with a Tanzanian study conducted by Mtega at al. (6). The highest age group affected in this study was second to fourth decade, a finding that is in agreement with other studies $(4,5,7)$. The study showed a male preponderance for the infections (1.4:1 male to female ratio), consistent with studies conducted in Nigeria and Uganda where males were more affected by orofacial bacterial infections. This could be because women are reported to have better 
oral health as they are more conscious of their oral health and seek oral health care more frequently than men $(4,8)$. Most patients suffering from advanced orofacial infections had informal employment, as is the case for most Kenyan citizens. Informal employment may be a reflection of low financial income. This limits the patients' oral health-seeking behavior from dental clinics and hospitals during early stages of the disease. Patients of low socioeconomic status stand the risk of poor nutrition, which may result in impaired host defense mechanism against infections $(5,7)$. The incidence of advanced infections such as Ludwig's angina has reduced in Africa due to introduction of antibiotics and early dental treatment. These advances are however still inaccessible to marginalized groups and patients with low socioeconomic status (9).

Swelling, pain and trismus were the most common presenting signs and symptoms in this study, consistent with a study conducted on a Nigerian population (7). Infections trigger host inflammatory response that results in pain and swelling, which are some of the signs of inflammation alongside redness, local heat and loss of function. Swelling of the tissues around the spaces in the floor of the mouth or larynx is dangerous as it can lead to respiratory embarrassment. Trismus ensues as a result of spread of infection to masticator space. Approximately $14.50 \%$ and $17.80 \%$ of the patients presented with dysphagia and respiratory distress respectively. Dysphagia and respiratory distress are most likely to occur as a result of pharyngeal, laryngeal and supraglotic edema and call for close patient monitoring for signs of upper airway embarrassment (10).

Odontogenic sources were the most common, as shown in previous studies where odontogenic infections were more common than non-odontogenic infections $(4,11,12,13)$. Permanent teeth were the most commonly involved with mandibular posterior teeth being the most commonly involved, consistent with results from previous studies $(4,5,11)$. Due to their large surface area, posterior teeth are subjected to most occlusal stresses, which can lead to micro/macro trauma to the soft tissues, stagnation of food debris, and decreased accessibility for proper oral hygiene practices such as brushing and flossing, hence caries $(4,7)$.

The submandibular space was the most commonly involved fascial space, consistent with previous studies where it was the most commonly affected fascial space $(7,12)$. However, a study on an Iranian population reported buccal space as the most commonly involved space in single space abscesses (11). In our study, the mandibular posterior teeth were the most commonly involved teeth. Infections involving the mandibular molars and premolars commonly involve the submandibular or sublingual spaces when the infection perforates the lingual plate of the mandible (14).

Odontogenic infections often involve more than one anatomic space, indicating that the fascial spaces in the head and neck are interconnected and the infections can rapidly spread contiguously from one space to another. Often, infections spread from the mandible or the maxilla into the sublingual, submandibular or masticator space to involve the parapharyngeal spaces (12). Bilateral involvement of the submandibular and sublingual spaces results in a condition called Ludwig's angina, which was the most common clinical diagnosis in this study, next was submandibular abscess.

Most patients did not have an accompanying systemic comorbidity. Diabetes mellitus and hypertension were the most common medical comorbidities and were often uncontrolled at the time of presentation. Diabetes mellitus has been reported to be the most common underlying systemic disease in patients who develop life-threatening complications from orofacial bacterial infections $(7,15,16)$. This finding was confirmed in our study: nine patients had diabetes mellitus and only two had a good outcome and seven died. Diabetic patients are not only at a high risk of developing infectious diseases, but also respond poorly to infections once they occur (16).

Treatment of orofacial bacterial infections involves removal of the cause of infection, incision and drainage to rid the body off purulent material and decompress the tissues, and antibiotic therapy $(7,13,14)$. Empirical antibiotic therapy is advocated for patients with orofacial bacterial infections before culture and sensitivity results. The most frequently prescribed 
antibiotics were Amoxicillin, and clavulanic acid with metronidazole, and ceftriaxone with metronidazole (Table 4). This was in agreement with a study by Ibiyemi in a Nigerian population and Pourdanesh in an Iranian population $(7,11)$.

Penicillin is the drug of choice in odontogenic infections as it is effective against most oral micro-organisms and has minimal side effects except allergic reactions. Penicillin is also comparatively cheaper than other antibiotics. However, resistance has been reported against penicillins (17). Addition of a beta lactamase inhibitor such as clavulanic acid confers resistance against beta lactamases (18). Our findings on emerging resistance to penicillins are consistent with a study by Akinkunmi et al., which described sensitivity to orofacial bacteria to antibiotic agents, where up to $98 \%$ beta-lactamase resistance was observed (19).

Metronidazole is also effective and it has activity against anaerobic organisms. It is often used in combination with a penicillin, cephalosporin or clindamycin $(5,17)$. Clindamycin was prescribed in $13.2 \%$ of the cases. It is a useful broad-spectrum antibiotic indicated when there is an allergy to penicillin (11). As antibiotic resistance grows, more complex and combination medications are required for management of orofacial infections. In the developing countries within sub-Saharan Africa, the threat of widespread resistance is due to lack of medical/dental services, and inadequate control of pharmaceutical products by the relevant government agencies $(20,21)$.

In other pharmacologic interventions provided, dexamethasone was the most prescribed in $62.7 \%$ $(n=74)$ of patients. The use of a corticosteroids in the management of these infections could be justified by their anti-inflammatory properties. However, corticosteroids are potentially immunosuppressive. Their use should be advocated when the benefits outweigh their immunosuppressive potential in the management of orofacial infections.

The most common surgical intervention was surgical incision and drainage (93.00\%), which was in most cases conducted with antibiotic therapy as an adjunct. Patients with abscesses as well as those with cellulitis underwent surgical incision and drainage. Incision and drainage rid the body of toxic purulent material and also decompress the tissues. This allows better perfusion of blood that contain antibiotics and other defensive elements, and increased oxygen, promoting quick clearance of infection (14).

The source of infection should be identified and eliminated either by extraction of the involved tooth or endodontic therapy when a tooth is the primary source of infection. About $42.2 \%$ of the patients had the involved tooth extracted while only $0.7 \%$ patients had endodontic therapy. It is important to identify the primary source of infection especially when it is of odontogenic source. In this study the source of infection was unspecified in $19.2 \%$ of the cases (Figure 2). Imaging plays an important role in identifying the source of infection and the anatomic spaces involved in the infection. This information is useful especially for surgical management of complicated orofacial infections. Both computerized tomography and magnetic resonance imaging provide reliable information on fascial spaces involved in the infection (22).

The treatment outcome was good in most patients. However, there was $7 \%$ mortality from the infections. This rate is higher than rates reported in a similar Ghanaian study by Blankson et al., which reported a $5.8 \%$ fatality rate (23). Late presentation was a common factor in both the Kenyan and Ghanaian fatalities. Patients presenting with comorbidities and underlying systemic conditions such as uncontrolled diabetes mellitus recorded higher levels of fatality than those with good general health. Because orofacial infections are managed empirically in most cases, the information derived from this study provides trends that may inform good clinical practices.

\section{Conclusion}

Orofacial bacterial infections can occur among all sociodemographic groups. The most common source of orofacial bacterial infection was odontogenic in nature. Orofacial bacterial infections are potentially lifethreatening if not diagnosed early and managed promptly. Early intervention is required for dental diseases so as to prevent advanced orofacial infections. 
Multidisciplinary teams are required to manage the severe morbidity and mortality of advanced orofacial infections.

\section{References}

1. Ghom A, Mhaske S. 2013. Textbook of oral pathology. 2nd ed. New Delhi: Jaypee Brothers Medical Publishers (P) LTD: 440 p.

2. Dahlén G. Non-odontogenic infections in dentistry. Periodontol 2000. 2009; 49:7-12.

3. Fragiskos FD. 2010. Oral surgery, $1^{\text {st }}$ ed. Berlin: Springer. 205 p.

4. Kudiyirickal MG, Hollinshead F. Clinical profile of orofacial infections: An experience from two primary care dental practices. Med Oral Patol Oral Cir Bucal. 2012; 17(4):533-7.

5. Kityamuwesi R, Muwaz L, Kasangaki A, et al. Characteristics of pyogenic odontogenic infection in patients attending Mulago Hospital, Uganda: a cross-sectional study. BMC Microbiol. 2015; 15:46-56.

6. Mtenga AA, Kalyanyama BM, Owibingire SS, et al. Cervicofacial necrotizing fasciitis among patients attending the Muhimbili National Hospital, Dar es Salaam, Tanzania. BMC Infect Dis. 2019; 19(1):642-649.

7. Ibiyemi ST, Okoje-Adesomoju VN, Dada-Adegbola, et al. Pattern of orofacial bacterial infections in a tertiary hospital in Southwest Nigeria. J West Afr Coll Surg. 2014; 4(4):112-141.

8. Akinbami BO, Akadiri O, Gbujie DC. Spread of odontogenic infection in Port Harcourt. Niger J Oral Maxillofac Surg. 2010; 68:2472-2477.

9. Okoje VN, Ambeke OO, Gbolahan OO. Ludwig's angina: An analysis of cases seen at the university college hospital, Ibadan. Ann Ib Postgrad Med. 2018; 16(1):61-68.

10. Mathew GC, Ranganathan LK, Gandhi S, et al. Odontogenic maxillofacial space infections at a tertiary care center in North India: a five-year retrospective study. Int $\mathrm{J}$ Infect Dis. 2012;16(4):296-302.

11. Pourdanesh F, Dehghani N, Azarsina M, et al. Pattern of odontogenic infections at a tertiary hospital in Tehran, Iran: a 10-year retrospective study of 310 patients. J Dent (Tehran). 2013; 10(4):319-328.
12. Han X, An J, Zhang Y, et al. Risk factors for lifethreatening complications of maxillofacial space infection. J Craniofac Surg. 2016; 27(2):385-390.

13. Uluibau I, Jaunay $\mathrm{T}$, Goss A. Severe odontogenic infections. Aust Dent J. 2005; 50:74-81.

14. Topazian RG, Goldberg MH, Hupp JR. 2002. Oral and maxillofacial infections, 4th ed. Philadelphia: WB Saunders. $158 \mathrm{p}$.

15. Zhang $\mathrm{C}$, Tang $\mathrm{Y}$, Zheng $\mathrm{M}$, et al. Maxillofacial space infection experience in West China: a retrospective study of 212 cases. Int J Infect Dis. 2010; 14:414-417.

16. Casqueiro J, Casqueiro J, Alves C. Infections in patients with diabetes mellitus: A review of pathogenesis. Indian $\mathrm{J}$ Endocrinol Metab. 2012; 16(1):27-36.

17. Bhagania M, Youseff $\mathrm{W}$, Mehra $\mathrm{P}$, et al. Treatment of odontogenic infections: An analysis of two antibiotic regimens. J Oral Biol Craniofac Res. 2018; 8(2):78-81.

18. Adamson OO, Adeyemi MO, Gbotolorun OM, et al. Comparison of sensitivity of bacteria isolated in odontogenic infections to ceftriaxone and amoxicillinclavulanate. Afr Health Sci. 2019; 19(3):2414-2420.

19. Akinkunmi EO, Adesunkanmi AR, Lamikanra A. Pattern of pathogens from surgical wound infections in a Nigerian hospital and their antimicrobial susceptibility profiles. Afr Health Sci. 2014; 14(4):802-809.

20. Barasa GB, Butt F, Onyango JF, et al. Antibiotic sensitivity patterns of aerobic bacterial agents in post-surgical orofacial infections. Ann Afr Surg. 2015; 12(1):32-37

21. Anyanechi C, Saheeb B. Toothache and self-medication practices: a study of patients attending a Niger delta tertiary hospital in Nigeria. Ann Med Health Sci Res. 2014; 4(6):884-888.

22. Thakur GVD. Masticator space abscess: a case report. IOSR J Dental and Medical Sciences. 2013: 7(2):64-67.

23. Blankson PK, Parkins G, Boamah MO, et al. Severe odontogenic infections: a 5-year review of a major referral hospital in Ghana. Pan Afr Med J. 2019; 32:71. 